\title{
Author Correction: Radiotherapy as a tool to elicit clinically actionable signalling pathways in cancer
}

\author{
Giulia Petroni, Lewis C. Cantley (1D, Laura Santambrogio, Silvia C. Formenti(i) and Lorenzo Galluzzi (i)
}

Correction to: Nature Reviews Clinical Oncology https://doi.org/10.1038/s41571-021-00579-w, published online 24 November 2021.

The authors failed to explicitly recognize the major experimental and conceptual input of Dr. C. Norman Coleman and collaborators to the development of the therapeutic paradigm discussed in this review. The authors apologize to Dr. Coleman, his colleagues and the entire field of radiation oncology and have rectified the final section of the introduction to reflect this contribution. This omission has been corrected in both the HTML and PDF versions of this manuscript.

https://doi.org/10.1038/s41571-022-00611-7 I Published online 18 February 2022

(c) Springer Nature Limited 2022 